\title{
COVID-19 Associated Refractory Immune Thrombocytopenia: A Case Report
}

Pusem Patir', [MD]
ORCID: 0000-0001-5201-4680

'Department of Hematology, Aydin State Hospital, Aydin, Turkey.

\section{mel ABSTRACT Clen}

We report here the patient with refractory immune thrombocytopenia associated with COVID-19 infection.

Keywords: COVID-19, immune thrombocytopenia

Corresponding Author: Pusem Patir

Aydin State Hospital, Department of Hematology, 09100, Aydin, Turkey.

Phone: +902562139002 / 2152

E-mail: pusemp@yahoo.com

https://doi.org/10.32552/2021.ActaMedica.556

Received: 16 January 2021, Accepted: 3 June 2021,

Published online: 5 September 2021

\section{INTRODUCTION}

Thrombocytopenia in coronavirus disease patients is a risk factor for increased morbidity and mortality [1]. Thrombocytopenia in COVID-19 patients may occur due to disseminated intravascular coagulation (DIC), sepsis, hemophagocytic lymphohistiocytosis (especially in pediatric patients) or medication. A single case study recently indicated that COVID-19 infection could be associated with immune thrombocytopenia (ITP) [2]. ITP is a rare autoimmune disease that increases the risk of bleeding, characterized by a platelet count of $<100 \times 10^{9} / \mathrm{L}$ [3]. Various risk factors, including environmental (e.g. infection, malignancy and drugs) and genetic predisposition, were identified for ITP. We present the case of refractory ITP associated with infection with COVID-19 that rituximab treatment benefited after the acute infection period of the COVID-19.

\section{CASE PRESENTATION}

A 72-year-old male presented with complaints of petechiae, oral mucosal hemorrhage and hematuria. Oropharyngeal swab PCR examination was performed due to fever, shortness of breath and cough 10 days before admission and COVID-19 infection was found positive. Platelet value at admission was $9 \times 10^{9} / \mathrm{L}$. In peripheral smear, platelet was less than $10 \times 10^{\%} / \mathrm{L}$ and schistocyte was not observed. The patient was diagnosed with COVID19-associated ITP after the exclusion of other causes of thrombocytopenia, including DIC, bacterial sepsis, and medication. Methylprednisolone 1 $\mathrm{mg} / \mathrm{kg} /$ day was started to the patient who did not respond to platelet transfusion. The patient, who was unresponsive to methylprednisolone treatment and whose platelet value was $1 \times 10^{9} / \mathrm{L}$ and whose hematuria continued, was administered intravenous immunoglobulin (IVIG) with a total dose of $2 \mathrm{~g} / \mathrm{kg}$ on the 4th day of admission. On the 11th day of admission, the patient was still unresponsive and whose thrombocyte value was $1 \times 10^{9} / \mathrm{L}$, no mass lesion was detected in the whole body computed tomography, and an increase in the number of megakaryocytes in the bone marrow with the tendency to cluster was observed. Eltrombopag $50 \mathrm{mg} /$ day was started. For 4 weeks, rituximab $375 \mathrm{mg} / \mathrm{kg} /$ week was administered to 
a patient whose thrombocyte value at control continued to be $1 \times 10^{9} / \mathrm{L}$ two weeks later. During the four-week follow-up period, the patient's thrombocyte value increased to $103 \times 10^{9} / \mathrm{L}$.

\section{DISCUSSION}

Viralinfections are associated with ITP [4]. We present the case of refractory ITP during active infection with COVID-19 here. Diagnosis of COVID-19 associated ITP can be difficult due to several potential causes, such as clot activation due to COVID-19 infection and subsequent thrombocytopenia. In addition, thrombocytopenia can also be caused by COVID-19 therapies containing heparin, azithromycin and hydroxychloroquine.

If COVID-19 patients are complicated by ITPassociated severe thrombocytopenia, IVIG may be the preferred first-line treatment option for faster platelet recovery compared to corticosteroids. Also, as IVIG inhibits the phagocytic abilities of macrophages [4], it may also be successful in treating COVID-19 infection in the early stages of COVID-19 [5]. Despite this, eltrombopag treatment was initiated in our patient because of inadequate response to steroid and IVIG. Even though there is minimal ITP data from COVID-19, mimetic thrombopoietin may be useful [6]. However, as treatment with thrombopoietin mimetics has been shown to increase the risk of venous thromboembolism in selected cases
[7], it should be used with caution in COVID-19 infection. Rituximab treatment was initiated due to the risk of exacerbation of coagulation activation by Eltombopag with COVID-19 infection and our patient's advanced age, and no response was obtained for 2 weeks. The safety of rituximab in the context of COVID-19 is uncertain. Rituximab, may compromise antiviral immunity due to $B$ cell depletion, increase the risk of re-infection and impair vaccine efficacy. In our case, development of severe acute respiratory syndrome due to COVID-19 or reactivation of COVID-19 was not observed during rituximab treatment and it was administered safely.

As a result, ITP is a serious complication of COVID-19 infection and it is important to be aware. ITP treatment choice should be based on balancing the risk of bleeding from ITP against the potential worsening of COVID-19 infection due to immunosuppressive therapy.

\section{CONFLICT of INTEREST}

The authors declare that they have no conflict of interest.

\section{INFORMED CONSENT}

Informed signed written consent was taken from the patient involved.
[1] Liu Y, Sun W, Guo Y, et al. Association between platelet parameters and mortality in coronavirus disease 2019: Retrospective cohort study. Platelets. 2020; 31(4): 490-496.

[2] Zulfiqar AA, Lorenzo-Villalba N, Hassler $P$, et al. Immune thrombocytopenic purpura in a patient with COVID-19. N Engl J Med. 2020; 382(18): e43.

[3] Cooper N, Ghanima W. Immune Thrombocytopenia. N Engl J Med. 2019; 381: 945-955.

[4] Swinkels M, Rijkers M, Voorberg J, et al. Emerging Concepts in Immune Thrombocytopenia. Front Immunol. 2018; 9: 880.
[5] Cao W, Liu X, Bai T, et al. High-Dose Intravenous Immunoglobulin as a Therapeutic Option for Deteriorating Patients With Coronavirus Disease 2019. Open Forum Infect Dis. 2020; 7(3): ofaa102.

[6] Bhattacharjee S, Banerjee M. Immune Thrombocytopenia Secondary to COVID-19: a Systematic Review. SN Compr Clin Med. 2020; 19: 1-11.

[7] Jansen AJG, Swart RM, te Boekhorst PAW. Thrombopoietinreceptor agonists for immunethrombocytopenia. N Engl J Med. 2011; 365: 2240-2241. 